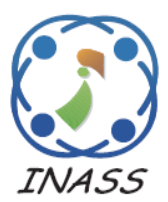

\title{
Dynamic Power Efficient Resource Allocation for 802.16 WiMAX Networks
}

\author{
Gopalan Meyyappan $^{1 *} \quad$ Marthandan Govindan $^{1} \quad$ Eswaran Chikkannan $^{1}$ \\ ${ }^{1}$ Multimedia University, Persiaran Mulitmedia, Cyberjaya, Malaysia \\ * Corresponding author's Email: gopalanmeyyappan@gmail.com
}

\begin{abstract}
In our previous work we have proposed a fuzzy based scheduling technique for multiple service flows in 802.16 WiMAX. This technique involves two entities namely evaluator and slot allocator. Where the evaluator arranges the real time and non real time service flows in two sub queues and then sorts them by their priority levels. The slot allocator obtains the priority information of service flows from the evaluator to allocate slots. For this work for we propose an extension which allocates the resources for the WiMAX Networks. This allocation is done based on the fairness of the node. During the allocation the approach considers the lifetime, transmission power, outrage probability and the power gain of the nodes. Based on these constraints the power and the bits are allocated to the source nodes in the network.
\end{abstract}

Keywords: WiMAX networks, Scheduling technique, Resource allocation.

\section{Introduction}

IEEE 802.16 WiMAX is signified as a standard for accessing wireless broadband networks. It is thought up by Institute of Electrical and Electronics Engineers (IEEE) to satisfy various end users requirements. It exploits Orthogonal Frequency Division Multiple Access (OFDMA) with the spectrum ranges between $1.25 \mathrm{MHz}$ and $28 \mathrm{MHz}$. It benefits both Time Division Duplexing (TDD) and Frequency Division Duplexing (FDD). Beam forming and Multiple Input Multiple Output (MIMO) are some of the sophisticated techniques used by WiMAX. Apart from this, it makes use of advanced coding techniques such as space-time coding and turbo coding.

WiMAX is extensively used in data, telecommunications (VoIP) and IPTV services (triple play). The bandwidth range of WiMAX makes available broadband connectivity through variety of devices to overall the world. WiMAX adds significant enhancements:

- It improves NLOS coverage by utilizing advanced antenna diversity schemes and hybrid automatic repeat request (HARQ).
- It adopts dense sub-channelization, thus increasing system gain and improving indoor penetration.

- It uses adaptive antenna system (AAS) and multiple input multiple output (MIMO) technologies to improve coverage.

- It introduces a downlink sub-channelization scheme, enabling better coverage and capacity trade-off.[1-4]

\subsection{Need for resource allocation}

WiMAX is one of the recent broadband wireless communication technologies, which supports resources allocation services. To increase the coverage and throughput, relay stations were introduced. To deal with the eventually increasing of the more traffic to compete with the limited QoS ad been a huge challenge. To handle these increasing traffics, WiMAX supports adaptive modulation and coding, such that the distance between the subscriber station (SS) and the base station (BS) determine the type of QoS to be used. Allocating radio resources dynamically in wireless network is highly complex and non-linear. It becomes even more complex when the wireless network is designed for heterogeneous traffics with different 
quality of service (QoS) requirement like WiMAX network. [3, 5-6]

\subsection{Advantages}

- By resource allocation it is possible to supply exactly required resources for the transmission by which the network's energy can be conserved.

- Through resource allocation management we can allocate the resources in an efficient manner and get the desired throughput.

\subsection{Issues}

Resources allocation is the important factor in the wireless networks, since the dynamic resource allocation, the allocation of the resources is dynamically changed as the channels change. The main aim of the resource allocator is the allocating the resources required for the transmission i.e., number of slots, for each user in each Mobile WiMAX frame. It is difficult to achieve the maximum throughput in the WiMax networks since each transmission demands different quality of services and this is possible to achieve only through the resource allocator in the networks. We require this resource allocator in order to use the resources in an efficient manner. [3, 5-7]

\subsection{Problem identification}

In the previous paper [1], we have proposed a fuzzy based scheduling technique for multiple service flows in 802.16 WiMAX. This technique involves two entities namely evaluator and slot allocator. When the service flow enters into the network, the evaluator distinguishes the service flow as real time or non real time. If the distinguished service flow belongs to real-time category then the evaluator estimates its priority using fuzzy logic technique applied over parameters such as delay, received signal strength and emergency degree. For the non real time service flows, the evaluator estimates its priority using fuzzy logic technique applied over parameters such as transmission rate and channel condition. Then the evaluator arranges the real time and non real time service flows in two sub queues and then sorts them by their priority levels. The slot allocator obtains the priority information of service flows from the evaluator to allocate slots. The drawback of this approach is that there is no technique approached here to allocate the resources required for the active transitions, because of which it is difficult to increase the throughput of the network.
To overcome the problems occurred in the previous work, we propose Dynamic fair multi-user resource allocation algorithm in this paper. the Dynamic fair multi-user resource allocation algorithm selects the privileged user based on some defined fairness criteria. Then it assigns the proper relays and sub-channels to the selected user. For these relays and the sub channels, the power and bits are allocated by the Dynamic fair multi-user resource allocation algorithm. Compared to the existing work, delivery ratio and throughput of our proposed are improved for both RT and NRT traffic.

Rest of this paper organized as follows. Section 2 relates some previous work with our work. Our proposed Dynamic power efficient resource allocation is presented in section 3. Results of our proposed approach are discussed in section 4 . Finally, this paper is concluded with section 5.

\section{Literature review}

J. Jin et al., [8] have proposed a novel cognitive WiMAX architecture with femto cells, where the base station and users are equipped with CRs and intelligently adjust power, channel, and other resources to accommodate the entire network ecosystem. In this new design, the authors have developed an optimization framework for location aware cooperative resource management, by jointly employing multi-hop cooperative communication, power control, channel assignment, primary user protection, buffer management, and fairness, and incorporating user, channel, and cooperative diversities. The main advantage of this approach is that the WiMAX achieves substantial performance improvement. However it is, energy efficiency of their proposed approach is not sufficient.

C. So-In et al., [7] have defined the Generalized Weighted Fairness (GWF) criterion that allows carriers to implement and apply either of the two fairness criteria to a Mobile WiMAX environment. Through this approach we can overcome the throughput fairness which is favorable to customers with a poor channel condition or those at a far distance. The temporal fairness will be preferred by the customers near the base stations and with good channel conditions can be achieved. The advantage of this approach is that it achieves a general weighted fair resource allocation in IEEE 802.16e Mobile WiMAX networks. Throughput of our proposed method is increased further than that of their proposed method.

T. A. Yahiya et al., [9] have proposed a frequency planning based on zone switching diversity scheme for multi cell OFDMA mobile 
WiMAX networks. This approach focuses on the use of Fractional Frequency Reuse (FFR) for guaranteeing the quality of service for the different service flows in the system. The proposed algorithm attempts to capture three types of diversity, namely, mutual interference diversity, traffic diversity, and selective fading channel diversity. The advantage is that the proposed algorithm for slot allocation makes a trade-off between maximizing overall throughput of the system while guaranteeing the Quality of Service $(\mathrm{QoS})$ requirements for a mixture of realtime and non-real-time service flows under different diversity configurations. They have allocated the resources in a hierarchical way so that delay of their proposed approach is increased.

J. Hajipour et al., [10] have proposed a dynamic distributed resource allocation algorithm. In this approach, where the BS and relays decide about the allocation of the power and sub-channels by passing messages among themselves and based on the local queue and channel state information. In this approach we study the dynamic allocation of power and sub-channels in an OFDMA downlink system with regenerative relays which have the capability of buffering the user's data to transmit in a suitable time. The advantage of this approach is that it improves the network's performance in terms of system throughput and users' queue stability. However it is, transmission power of their proposed method is increased.

S. M. Jafari et al., [11] have proposed a twophase Learning Automaton queuing (2PLAQ) algorithm tailored for uplink scheduling in the WiMAX network. This approach aims to strike the balance between delay requirement and fair bandwidth allocation. This approach has been designed to have an ability to accommodate integrated traffic in the networks with effective scheduling schemes. The advantage of the proposed approach is that it performs effectively to the integrated traffic composed of messages with or without time constraints and achieves proportional fairness among different types of traffic. Packet drop of our proposed method is reduced further than that of their proposed method.

H. Banizaman et al., [12] have investigated and analyzed the Dynamic fair multi-user resource allocation algorithm that aims at improving the allocation of resources in a dual hop OFDMA cooperative network consisting in multi sourcedestination pairs and multiple decode-and-forward relays. In this approach the algorithm actually based on some defined fairness criteria the privileged user is selected. For these privileged users the proper relays and sub-channels are assigned to the selected user in order to minimize its transmission power under the BER and data rate constraint based on the equivalent channel gains. The power and bits are allocated to the subchannels assigned to the selected user. The advantage of this approach is that it mainly concentrates on decreasing the transmission power of the network. Compared to this method, throughput of our proposed method is improved.

\section{Proposed solution}

\subsection{Overview}

To overcome the drawback mentioned above we are proposing a Dynamic fair multi-user resource allocation algorithm for the resource allocation in the network. Through this technique it is possible to allocate the resources for the nodes and the sub channels for the transmission.

In our proposal we are extending the previous work by implement the Dynamic fair multi-user resource allocation algorithm in the fuzzy based scheduling technique for multiple service flows in 802.16 WiMAX. Here in this proposal, based on the priorities found in the previous work, the Dynamic fair multi-user resource allocation algorithm selects the privileged user based on some defined fairness criteria. Then it assigns the proper relays and subchannels to the selected user. For these relays and the sub channels, the power and bits are allocated by the Dynamic fair multi-user resource allocation algorithm.

The advantage of this approach is that, through this technique it is possible to decrease the transmission power of the network. We get the desired maximum throughput from the network with the limited resources. And also we can conserve the resources since we are using an allocating technique and sleep scheduling mechanism.

\subsection{Dynamic fair resource allocation}

In this approach the dynamic fair resource allocation initially considers the network performance parameters like lifetime and outage probability in order to provide fairness in the network. In the network the source nodes may have quite disparate lifetimes, since they have different channel conditions, initial battery energy levels, QoS constraints, power allocation strategies and so on.

\subsubsection{Estimation of the lifetime}

The average network lifetime of the network is estimated which considers the lifetime total source 
nodes.

$$
L t=\frac{1}{S} \sum_{s=1}^{S} L t_{S}
$$

Where,

$L t$ is the average lifetime of the network, $S$ is the total number of source nodes and the $L t_{\mathrm{s}}$ is the lifetime of the source node.

The ratio between the maximum lifetimes and minimum lifetime of the source nodes in the network is adopted as an indicator for the lifetime fairness that is given in Eq. (2).

$$
\kappa_{l}=\frac{L t_{\min }}{L t_{\max }}
$$

Where,

$k_{l}$ is an indicator for the lifetime fairness, $L t_{\text {min }}$ minimum lifetime of the network and the $L t_{\max }$ is the maximum lifetime of the network.

$$
\kappa_{l}= \begin{cases}1, & \text { when the system achieves the } \\ & \text { great fairness in which } \\ & \text { all users would achieve } \\ & \text { the same lifetime. } \\ & \text { when severe unfairness occurs, } \\ & \text { as some source nodes run } \\ & \text { out of energy very quickly. }\end{cases}
$$

\subsubsection{Estimation of the outage probability}

An outage occurs in the network when the total required transmission power for transmitting one OFDM block, is greater than maximum power. Since an alternative network performance parameter is sources outage probability.

$$
o p_{\text {out }, s}=\operatorname{prob}\left(\sum_{n=1}^{N} P_{s}^{n}>P_{\max }\right)
$$

Where,

$o p_{\text {outs }}$ is the outage probability of source node $s$, $n$ is the $n^{\text {th }}$ sub-channel, $P_{\max }$ be the maximum allowed power per user for transmitting one OFDM block, the $N$ is the total number of sub-channels in the network and $P_{s}{ }^{n}$ is defined as the amount of power transmitted on sub-channel $n$ if the subchannel is assigned to user $s$.

\subsubsection{Selection of source node}

The source node $(s)$ in the network is selected based on some of the conditions. Based on these conditions the source node is selected by the network. For the selected source node the power will be allocated based on the conditions verified below.

\section{Condition 1}

In case of minimization of the transmission power of the least considered source node, i.e. the source node which requires the highest transmission power or the source node with the poorest channel conditions. In such case, source nodes are treated with no consideration for their battery energy levels. Using this condition in the allocation algorithm provides identical outage probabilities among source nodes.

$$
s=\arg _{s} \min \left(P_{\max }-\sum_{n=1}^{N} P_{s}^{n}\right)
$$

\section{Condition 2}

Minimization of the transmission power of the least privileged user, i.e. the user which has the weakest remaining battery energy or the poorest channel conditions

$$
\begin{gathered}
s=\arg _{s} \min \left(E_{s}^{{ }_{s}}\right) \\
E_{s}^{{ }_{s}}=E_{S}-\Delta t \sum_{n=1}^{N} P_{s}^{n} \\
P_{S}^{n}= \begin{cases}\left(P_{D}\right)_{s, d}^{n}, & \text { if } \mathrm{n} \text { used in direct link } \\
\sum_{r=1}^{R}\left(P_{C}\right)_{s, r}^{n}, & \text { if } \mathrm{n} \text { used in cooperative link }\end{cases}
\end{gathered}
$$

Where,

$\left(P_{D}\right)^{n} s, d$ - The minimum power required for the direct transmission mode of source $m$ and subchannel $n,\left(P_{c}\right)^{n} s, r$ is the required source power to guarantee successful transmission from source node $s$ to relay node $r$ in sub-channel $n$ which is the first half of transmission, $\Delta t$ is the allocation period, $E_{s}$ is the estimation of the new battery energy level.

The minimum power required for the direct transmission mode of source $\mathrm{m}$ and sub- channel $n$ is given by the below Eq. (9),

$$
\left(P_{D}\right)_{s, d}^{n}=\frac{P_{r e q}\left(b_{s}^{n}\right)}{\left(P_{G}\right)_{s, d}^{n}}
$$


Where,

$P_{r e q}\left(b_{s}{ }^{n}\right)$ is the required received power in subchannel $\mathrm{n}$ for reliable reception $b_{s}{ }^{n}$ is the bits/symbol for user $s,\left(P_{G}\right)_{s, d}^{n}$ is the power gain between the source node $\mathrm{s}$ and the destination node $d$ in subchannel $n$.

The required received power in sub-channel $\mathrm{n}$ for reliable reception $b_{s}{ }^{n}$ is the bits/symbol for user $s$ is given by the below Eq. (10),

$$
P_{r e q}\left(b_{s}^{n}\right)=S N R \frac{N_{0} B}{N}
$$

Where,

$N_{0}$ is the power spectral density of the additive white Gaussian noise (AWGN) and $N_{0}$ is the same for all source nodes, relay nodes, and destination nodes and this $N_{o}$ is also assumed to be constant over all sub-channels. $B$ is the total system bandwidth. SNR is given by.

$$
S N R=\log \left(P_{t x}\right)-\log \left(P_{t x}\right) d B
$$

\section{Previous paper}

The total power used in cooperative link transmission. i.e. if the transmission has to take place through the relay node between the source node and the destination node is given by the below Eq. (12),

$$
\left(P_{C}\right)_{s, r, d}^{n}=\left(P_{C}\right)_{s, r}^{n}+\left(P_{C}\right)_{r, d}^{n}
$$

The minimum power required for cooperative link transmission using sub-channel $n$ at source $s$ and relay node $r$ is given by the below equation Eq. (13),

$$
\left(P_{C}\right)_{s, r, d}^{n}=\frac{P_{r e q}\left(b_{s}^{n}\right)}{\left(P_{G}\right)_{s, r, d}^{n}}
$$

\section{Condition 3}

$$
P_{\max }-\sum_{n=1}^{N} P_{s}^{n}<0
$$

This above condition will be implemented when the combination of conditions 1 and 2 in order to equalize both outage probability and lifetime of source node, According to this criterion, if there exists a source node. If the above condition is true than the condition 1 will be used otherwise we use condition 2.

\subsubsection{Estimation of power gain}

The total power gain in the network an equivalent channel power gain of source node $s$ in sub-channel $\mathrm{n}$ through relay node $\mathrm{r}$ is given below equation. The estimation of the total power gain considers the transmission power and the transmitter gain from the previous paper.

$$
\left(P_{G}\right)_{s, r, d}^{n}=\Delta_{s}^{n} \alpha_{s, d}^{n}+\sum_{r=1}^{R} \rho_{s, r}^{n}\left(P_{t_{x}}\right)_{s, r, d}^{n}
$$

Where,

$\left(P_{G}\right)_{s, r, d}^{n}$ is the total power gain between the source and destination nodes through relay node $r$,

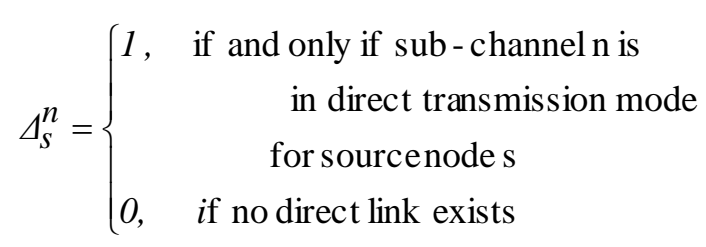

$$
\rho_{s, r}^{n}=\left\{\begin{array}{cc}
1, & \text { if more than } 1 \text { subchannel used } \\
& \text { for transmiossion } \\
0, & \text { if subchannels used is } \leq 1
\end{array}\right.
$$

$A$ and $P_{t x}$ are the transmitter gain and the power transmission which are considered from the previous paper (i.e. which are used in the Eq. (2))

\subsubsection{Algorithm for power and bits allocation}

Step 1: $b_{s}^{n}=0, \forall n \in n(s)$

Step 2: $\Delta P_{s}^{n}=\frac{P_{r e q}(1)}{\left(P_{G}\right)_{s, r, d}^{n}}-\frac{P_{r e q}(0)}{\left(P_{G}\right)_{s, r, d}^{n}}, \forall n \in n(s)$

Step 3: while $\sum_{n \in n(s)} b_{s}^{n} \leq R_{s}$

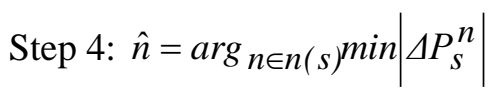

Step 5: $b_{s}^{\hat{n}}=b_{s}^{\hat{n}}+1$

Step 6: $\Delta P_{s}^{\hat{n}}=\frac{P_{r e q}\left(b_{s}^{\hat{n}}+1\right)}{\left(P_{G}\right)_{s, r, d}^{\hat{n}}}-\frac{P_{r e q}\left(b_{s}^{\hat{n}}\right)}{\left(P_{G}\right)_{s, r, d}^{\hat{n}}}$

Step 7: end while

Step 8: for all nen(s)

Step 9: $P_{s}^{n}=\frac{P_{r e q}\left(b_{s}^{n}\right)}{\left(P_{G}\right)_{s, r, d}^{n}}$ 
Step 10: end for

During the allocation of the bits to the nodes in the network, during this allocation initially all the sub channels are considered. These assigned sub channels to the source node are checked with the sub channels in the network. For those sub-channels which are assigned to the source node are assigned with the bits for the transmission.

$$
\left.\sum_{n \in n(s)} b_{s}^{n}=R_{s}\right)
$$

$R_{s}$ is the target data rate of user $s$ (bits per OFDM symbol) and $b_{s}{ }^{n}$ be the number of bits assigned to source sub-channel $n$ of user $s$.

The required total transmission power is computed for the source node $s$ before any subchannel is allocated. The most favorable relay and sub-channel are then assigned to mc based on the equivalent channel gains i.e. which is estimated by the Eq. (14), and the new total transmission power is calculated after the run of single source node.

$$
P_{s}^{\text {new }}=\sum_{n \in n(s)} P_{s}^{n}
$$

In the network in any case if the transmission power of the source node could not be decreased any more, then that source node would be removed from the set of source nodes.

$$
\begin{gathered}
P_{S}^{\text {new }}-P_{S}^{\text {old }} \geq 0 \\
S_{T}=S_{T}-\{s\}
\end{gathered}
$$

Where,

$S_{T}$ is the set of users whose transmission power can still be reduced.

\subsection{Algorithm of the whole approach}

Step 1: selection of user based on the fairness criteria

Step 2: calculate the total transmission power required by the source node $s$

Step 3: relay nodes and the assignment of the subchannels for the source nodes

Step 4: calculate the equivalent channel power gain i.e. according to the Eq. (15)

Step 5: Allocation of the bits and power to the source nodes

Step 6: Allocate the bits to the source node according to the sub-channels assigned
Step 7: Calculate the total transmission power required for the source nodes based on the subchannels assigned for the power allocation

Step 8: Check whether the transmission power could be decreased of the source node, if it can be decreased then that particular source node will be removed.

\subsection{Flowchart}

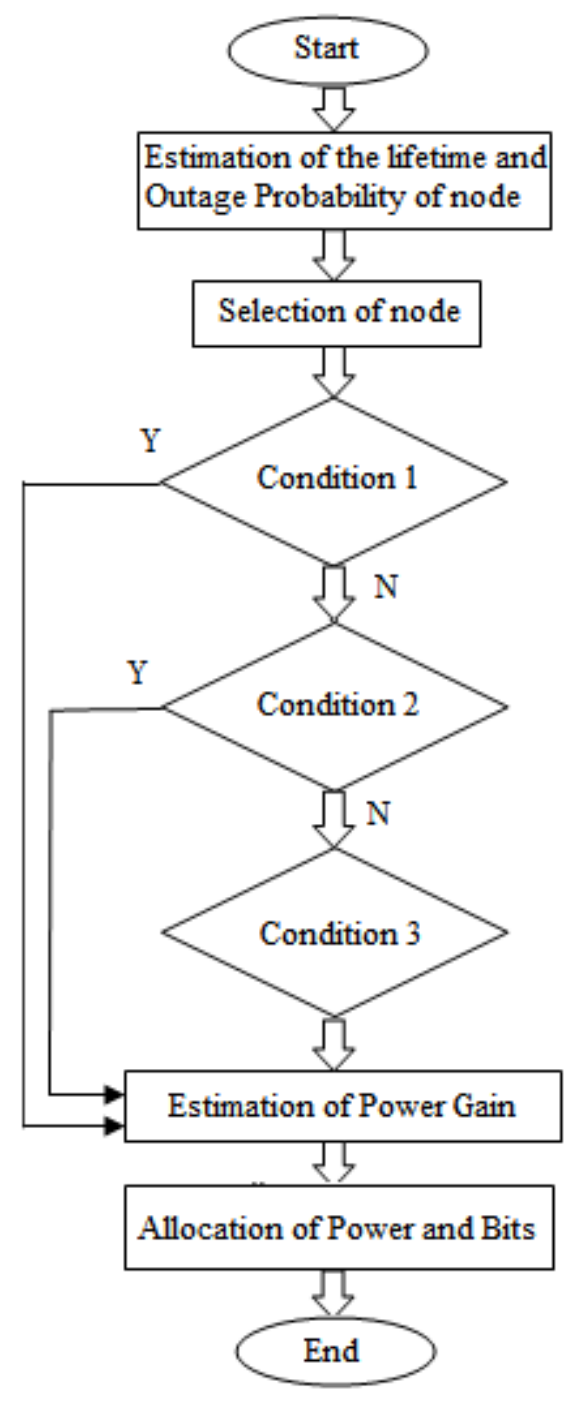

Figure.1 Flowchart decribes the algorithm of whole approach

Fig. 1 shows the flowchart of the algorithm described in section 3.3. In this approach, initially the lifetime and the outrage probability of all the nodes in the network is calculated. In those nodes before selecting the source nodes the three conditions are checked. The node which satisfies any of the conditions, then its power gain will be estimated. According to the power gain of the source node the power and the bits will be allocated 
to the source nodes.

\section{Simulation results}

\subsection{Simulation model and parameters}

Network simulator (NS2) is used evaluate performance of the proposed Load Based Sleep Scheduling Technique (LBSS). The proposed scheme is implemented over IEEE 802.16 MAC protocol. The simulation settings and parameters are summarized in table 1.

In the simulation, clients (SS) and the base station (BS) are deployed in a 1000 meter x 1000 meter region for 50 seconds simulation time. All nodes have the same transmission range of 500 meters. In the simulation, both the CBR and Video traffic are used. CBR is used for non-real time traffic and Video is used for real-time traffic.

There are 8 downlink traffic flows from BS to SS. Among the 8 flows, 4 CBR and 4 Video flows are used.

Table 1. Simulation Settings

\begin{tabular}{|l|l|}
\hline Area Size & $1000 \mathrm{X} 1000$ \\
\hline Mac & 802.16 \\
\hline Clients & 10 \\
\hline Radio Range & $500 \mathrm{~m}$ \\
\hline Simulation Time & $50 \mathrm{sec}$ \\
\hline Routing Protocol & DSDV \\
\hline Real-time Traffic & Video \\
\hline $\begin{array}{l}\text { Non real-time } \\
\text { traffic Exponential }\end{array}$ & \\
\hline Physical Layer & OFDM \\
\hline Video Trace File & JurassikH263- \\
& $256 \mathrm{k}$ trace.dat \\
\hline Channel Error Rate & 0.01 \\
\hline Packet Size & 1500 bytes \\
\hline Frame Duration & 0.005 \\
\hline Transmission Rate & $250 \mathrm{~Kb}, 500 \mathrm{~Kb}, 750 \mathrm{~Kb}$, \\
& $1000 \mathrm{~Kb}, 1250 \mathrm{~Kb}$ \\
\hline Simulation time & $65 \mathrm{~seconds}$ \\
\hline
\end{tabular}

\subsection{Performance Metrics}

We compare our proposed Dynamic Power Efficient Resource Allocation (DPERA) scheme with the Dynamic Distributed Resource Allocation (DDRA) Scheme [10] scheme and Dynamic resource allocation using Particle Swarm Optimization (DPSO) [5]. We mainly evaluate the performance according to the following metrics:

Aggregated Throughput: We measure the received throughput (in $\mathrm{Mb} / \mathrm{s}$ ) for both traffic of all flows
Average end-to-end delay: The end-to-enddelay is averaged over all surviving data packets from the sources to the destinations.

Delivery Ratio: It is the number of packets successfully received by the receiver.

Residual Energy: It is the average residual energy of all the nodes during the transmission.

The parameters are extracted from the trace file. The performance results are presented in the next section.

\subsection{Results}

The transmission rate of both real-time (RT) and non real-time (NRT) is varied as $250,500,750$, $1000,1250 \mathrm{~Kb}$. There are 10 traffic requests containing both RT and NRT traffic.

The end-to-end delay for NRT and RT traffic flows are given in Fig. 2 and 3, respectively. In both figures, the delay linearly increases, when the traffic rate is increased, since more resources have to be allocated for the flows. For NRT traffic, packet delay of our proposed approach is reduced to $13 \%$ and $26 \%$ than that of DDRA and DPSO respectively. For RT traffic, packet delay of our proposed approach is reduced to $58 \%$ and $66 \%$ than that of DDRA and DPSO respectively.

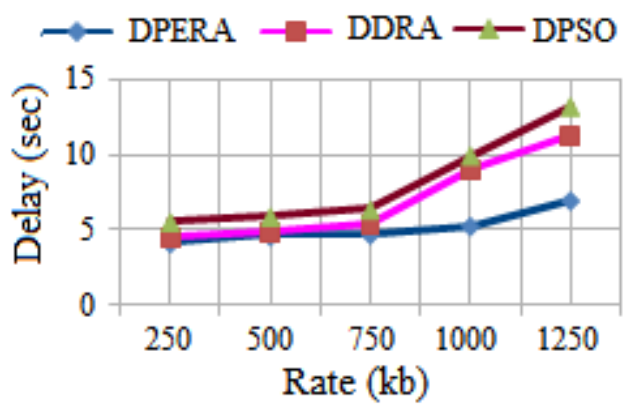

Figure.2 Rate Vs NRT-Delay

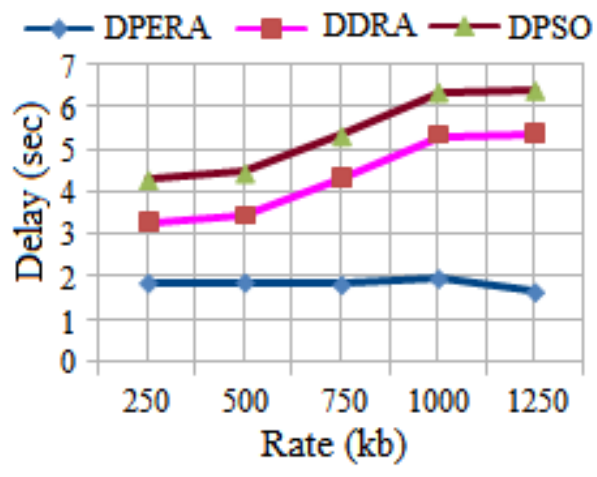

Figure.3 Rate Vs RT-Delay 


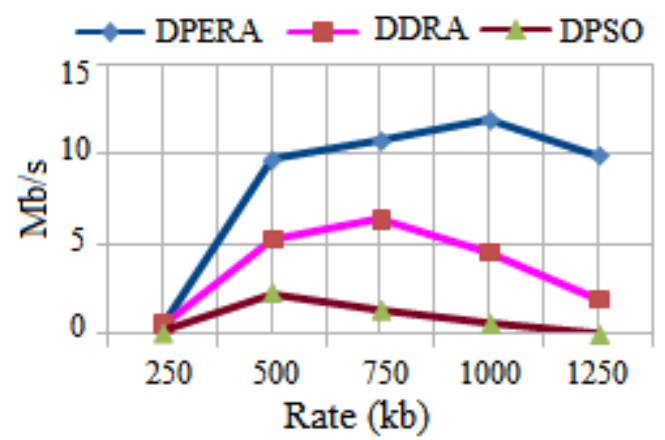

Figure.4 Rate Vs NRT-Throughput

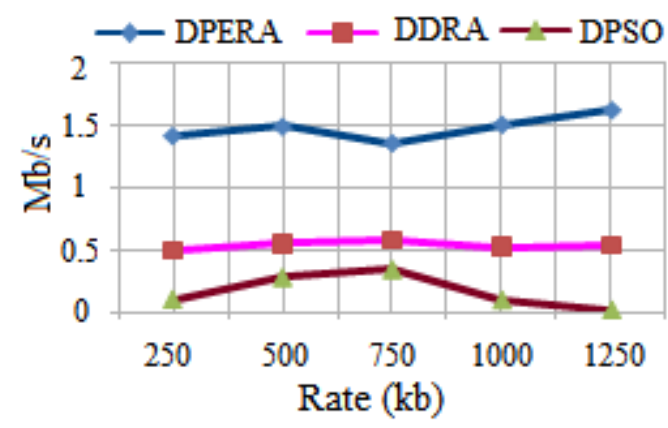

Figure.5 Rate Vs RT-Throughput

The received throughput for NRT and RT traffic flows are given in Fig. 4 and 5, respectively.

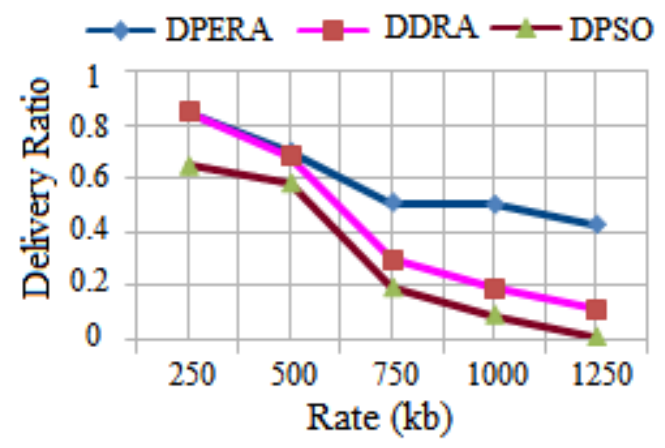

Figure.6 Rate Vs NRT-Delivery ratio

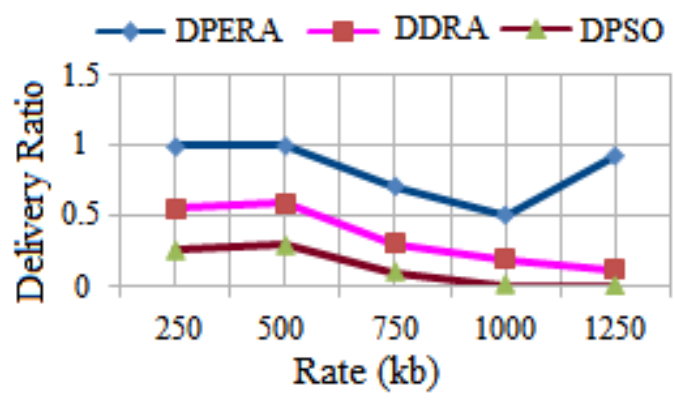

Figure.7 Rate Vs RT-Delivery ratio

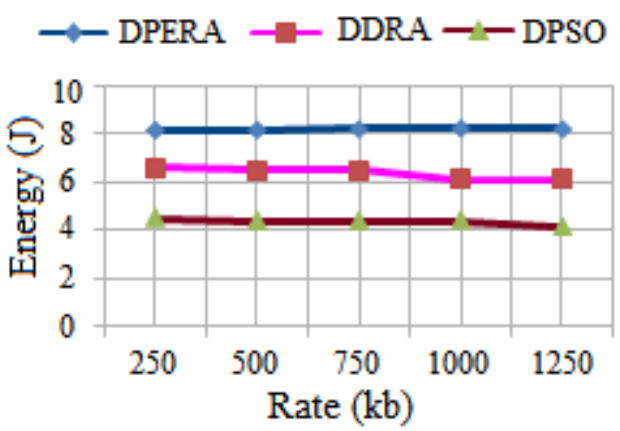

Figure.8 Rate Vs Residual Energy

From the Fig. 4 and 5 we can see that, in NRT traffic, the throughput is increasing whereas in RT traffic, it remains constant. We can see that DPERA attained higher throughput when compared to DDRA in both cases. Compared to the DDRA and DPSO, throughput of our proposed approach is increased to $68 \%$ and $96 \%$ respectively for NRT traffic. For RT traffic, throughput of our proposed approach is increased to $85 \%$ and $98 \%$ than that of DDRA and DPSO.

The measured packet delivery ratio of NRT and RT traffic flows are given in Fig. 6 and 7, respectively. From the figures we can see that, the packet delivery ratio tends to decrease as the rate increases. This because of the fact that there will be more packet drops due to outage probability. We can see that DPERA attained higher delivery ratio when compared to DDRA in both cases. For NRT traffic, delivery ratio of our proposed approach is increased to $79 \%$ and $96 \%$ than that of DDRA and DPSO respectively. For RT traffic, delivery ratio of our proposed approach is increased to $15 \% \%$ and $75 \%$ than that of DDRA and DPSO respectively.

The average residual energy in case of both RT and NRT traffic is illustrated in Fig. 8. From the figure we can see that DPERA has slightly higher residual energy, than DDRA scheme. Residual energy of our proposed approach is increased to $27 \%$ and $87 \%$ than that of DPERA and DPSO respectively.

\section{Conclusion}

In our previous work we have proposed a fuzzy based scheduling technique for multiple service flows in 802.16 WiMAX. This technique involves two entities namely evaluator and slot allocator. Where the evaluator arranges the real time and non real time service flows in two sub queues and then sorts them by their priority levels. The slot allocator obtains the priority information of service flows from the evaluator to allocate slots. For this work for 
we propose an extension which allocates the resources for the WiMAX Networks. This allocation is done based on the fairness of the node. During the allocation the approach considers the lifetime, transmission power, outrage probability and the power gain of the nodes. Based on these constraints the power and the bits are allocated to the source nodes in the network. The advantage of this approach is that, through this technique it is possible to decrease the transmission power of the network. We get the desired maximum throughput from the network with the limited resources. Also we can conserve the resources since we are using an allocating technique and sleep scheduling mechanism. As future work, an optimized algorithm will be used for resource allocation.

\section{References}

[1] M. S. Arhaif, "Comparative Study of Scheduling Algorithms in WiMAX", International Journal of Scientific \& Engineering Research, Vol. 2, No. 2, pp. 1-7, 2011.

[2] C. So-In, R. Jain \& A. K. Tamimi, "Scheduling in IEEE 802.16e Mobile WiMAX Networks: Key Issues and a Survey", IEEE Journal on Selected Areas in Communications, Vol. 27, No. 2, pp. 156-171, 2009.

[3] C. So-In, R. Jain \& A. K. Al-Tamimi, "Resource Allocation in IEEE 802.16 Mobile WiMAX", Auerbach Publications, 2010.

[4] R. K. Jha, A. V. Wankhede \& U. D. Dalal, "A Survey of Mobile WiMAX IEEE 802.16m", International Journal of Computer Science and Information Security, Vol. 8, No. 1, pp. 125-131, 2010.

[5] D. S. Shursquo, S. S. Yusof \& N. Fisal, "Dynamic resource allocation in mobile WiMAX using particle swarm optimization techniques", International Journal of the
Physical Sciences, Vol. 6, No. 5, pp. 1009-1014, 2011.

[6] Y. Hua, Q. Zhang and Z. Niu, "Resouce allocation in multi-cell OFDMA-based Relay Networks", In INFOCOM, Proceedings IEEE, pp. 1-9, 2010.

[7] C. So-In, R. Jain \& A. K. Al-Tamimi, "Generalized Weighted Fairness and its Application for Resource Allocation in IEEE 802.16e Mobile WiMAX", Computer and Automation Engineering (ICCAE), The 2nd International Conference, Vol. 1, pp. 784-788, 2010.

[8] J. Jin and B. Li, "Cooperative Resource Management in Cognitive WiMAX with Femto Cells", Infocom, 2010 Proceedings IEEE, pp. 552-560, 2010

[9] T. Ali-Yahiya and H. Chaouchi, "Fractional Frequency Reuse for Hierarchical Resource Allocation in Mobile WiMAX Networks", EURASIP Journal on Wireless Communications and Networking, No. 1, pp. 363065, 2010.

[10] J. Hajipour, A. Mohamed and V. C. Leung, "Dynamic Distributed Resource Allocation in Relay Assisted OFDMA Networks", The Eighth International Conference on Wireless and Mobile Communications, pp. 415-420, 2012.

[11] S. M. Jafari, M. Taghipour and M. R. Meybodi, "Bandwidth Allocation in WiMAX Networks Using Learning Automaton", World Applied Sciences Journal, Vol. 15, No. 4, pp. 576-583, 2011.

[12] H. Banizaman and S. M. T. Almodarresi, "Dynamic resource allocation algorithm in multi-user cooperative OFDMA systems: considering QoS and fairness constraints", Springer, Wireless Networking, Vol. 18, No. 4, pp. 365-380, 2012. 